\title{
Análise espacial dos casos confirmados de Zika Vírus no estado de São Paulo, Brasil
}

\begin{abstract}
RESUMO | Objetivo: analisar a distribuição espacial dos casos confirmados de Zika Vírus. Método: Estudo epidemiológico, do tipo ecológico, exploratório, obtidos a partir dos casos confirmados de Zika Vírus, notificados no Sistema de Informação de Agravos de Notificação, ocorridos no Estado de São Paulo em 2016. Foi aplicada a técnica de georreferenciamento. A análise espacial foi feita a partir do estimador de densidade Kernel e a estatística de varredura fornecida pelo SaTScan ${ }^{\mathrm{TM}}$ version 9.6. Resultado: A análise de Kernel apontou áreas quentes na região do estudo. Por meio da estatística de varredura foram identificados os aglomerados dos Riscos Relativos dos 645 municípios. A distribuição espacial de Zika Vírus seguiu padrão heterogêneo, pois, os focos tenderam-se a agregar-se em áreas específicas. Os municípios das regiões norte e noroeste apresentaram crescente Risco Relativo. Conclusão: Este estudo oferece informações fundamentais para auxiliar gestores em saúde pública nas tomadas de decisões nos níveis municipal e estadual
\end{abstract}

Palavras-chaves: Zika Vírus; Infecção por Zika Vírus; Análise Espacial; Monitoramento Epidemiológico.

ABSTRACT | Objective: to analyze the spatial distribution of confirmed Zika Virus cases. Method: Ecological, exploratory, epidemiological study, obtained from confirmed cases of Zika Virus, reported in the Notifiable Diseases Information System, which occurred in the State of São Paulo in 2016. The georeferencing technique was applied. Spatial analysis was performed using the Kernel density estimator and the scan statistics provided by SaTScan TM version 9.6. Result: The Kernel analysis showed hot areas in the study region. Through the scanning statistics, the Relative Risks clusters of the 645 municipalities were identified. The spatial distribution of Zika Virus followed a heterogeneous pattern, as the outbreaks tended to aggregate in specific areas. The municipalities of the north and northwest regions presented an increasing Relative Risk. Conclusion: This study offers fundamental information to assist public health managers in decision-making at the municipal and state levels.

Keywords: Zika Virus; Zika Virus Infection; Spatial Analysis; Epidemiological Monitoring.

RESUMEN | Objetivo: analizar la distribución espacial de casos confirmados de Virus Zika. Método: Estudio epidemiológico, exploratorio y ecológico, obtenido de casos confirmados de Virus Zika, reportado en el Sistema de Información de Enfermedades de Notificación, que ocurrió en el Estado de São Paulo en 2016. Se aplicó la técnica de georreferenciación. El análisis espacial se realizó utilizando el estimador de densidad de Kernel y las estadísticas de escaneo proporcionadas por SaTScan TM versión 9.6. Resultado: el análisis de Kernel mostró áreas calientes en la región de estudio. A través de las estadísticas de escaneo, se identificaron los grupos de Riesgos relativos de los 645 municipios. La distribución espacial del virus Zika siguió un patrón heterogéneo, ya que los brotes tendieron a agregarse en áreas específicas. Los municipios de las regiones norte y noroeste presentaron un riesgo relativo creciente. Conclusión: Este estudio ofrece información fundamental para ayudar a los administradores de salud pública en la toma de decisiones a nivel municipal y estatal.

Palavras claves: Virus Zika; Infección por el virus del Zika; Análisis espacial; Monitoreo epidemiológico.

\section{Roudom Ferreira Moura}

Doutorando do Programa de Epidemiologia da Faculdade de Saúde Pública Universidade de São Paulo / Técnico do Centro de Vigilância Epidemiológica "Prof. Alexandre Vranjac" - Secretaria de Estado da Saúde de São Paulo / Professor da Universidade São Judas Tadeu / São Paulo.

\section{Aparecido Batista de Almeida}

Doutorando do Programa de Epidemiologia da Faculdade de Saúde Pública - Universidade de São Paulo / Técnico do Centro de Vigilância Epidemiológica "Prof. Alexandre Vranjac" Secretaria de Estado da Saúde de São Paulo.

Recebido em: $31 / 03 / 2020$

Aprovado em: 28/04/2020
INTRODUÇÃO

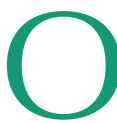

Zika Vírus é uma enfermidade viral, de evolução benigna, caracterizada pelo quadro clínico de febre baixa, exantema maculopapular pruriginoso, hiperemia conjuntival não pruriginosa e não purulenta, artralgia ou artrite, dores musculares, cefaleia e manifestações digestivas ${ }^{1}$. No entanto, a infecção pelo Vírus Zika pode causar microcefalia em recém-nascidos, Síndrome de Guilan-Barré e óbito².

No segundo semestre de 2014, suspeitas de Zika Vírus foram registradas em algumas cidades do Nordeste Brasileiro e confirmadas, em maio de 2015, pelo Ministério da Saúde do Bra- sil com transmissão autóctone $e^{3,4}$.

O Vírus Zika é um flavivírus emergente, transmitido, principalmente, pelo mosquito Aedes Aegypti que foi isolado pela primeira vez a partir de um macaco sentinela na floresta de Zika, em Uganda, em $1947^{5}$.

O mosquito Aedes Aegypti é um dos principais vetores do Vírus Zika para humanos em todo o mundo ${ }^{5}$. Esse vetor está disperso em grande parte do território brasileiro. Foi relatado por Nunes et al. que o mosquito Aedes Aegypti estava presente em 5172 dos 5494 municípios do Brasil no ano de $2013^{6}$.

O Vírus Zika foi introduzido no Brasil entre 2013 e 2015, provavelmente, importado do Pacífico, e causou surto epidemiológico que atingiu 
pico em novembro de 2015, espalhando-se rapidamente em todo o país ${ }^{7}$.

Nesse contexto, pesquisas com metodologias de análise espacial são relevantes para conhecer a realidade dos locais em que a doença se espaIhou, se possui casos alóctones e/ou autóctones, bem como avaliar a magnitude da doença, sendo fundamental para a realização de intervenções e efetivas ações para o seu controle, tendo em vista a história natural da doença e a determinação social do processo saúde-doença de modo a contribuir para promoção da saúde coletiva da população.

As técnicas de análise espacial tem sido utilizadas pela epidemiologia, pois, permitem a realização de estudos da distribuição espacial de agravos, doenças e situações de riscos, o que possibilita a detecção de áreas vulneráveis, conhecer mais detalhadamente os padrões das condições de saúde de uma população, bem como evidenciar disparidades ${ }^{8,9}$.

No Brasil, entre 2015 e 2016, anos dos surtos epidemiológicos de Zika Vírus, foi estimado que 65 milhões de pessoas viviam em áreas de alto risco para essa doença. As regiões sudeste e nordeste do Brasil apresentaram as maiores áreas de alto risco para Zika Vírus ${ }^{10}$.

No Estado de São Paulo, pesquisas sobre o Zika Vírus são necessárias porque se trata de uma doença emergente a partir de $2015^{11}$. Além disso, as notificações dos casos de Zika Vírus, no Brasil, passaram a ser obrigatória em fevereiro de $2016^{12}$ e ainda são poucos os estudos que realizaram análise espacial dessa doença no país ${ }^{10}$ e no Estado de São Paulo não foram encontradas pesquisas sobre essa temática.

Tendo em vista essas premissas, o presente estudo buscou respostas ao seguinte questionamento: como se deu a distribuição espacial do surto epidemiológico de Zika Vírus no Estado de São Paulo no ano de 2016? Para res- ponder a questão proposta, essa pesquisa teve como objetivo analisar a distribuição espacial dos casos confirmados de Zika Vírus no Estado de São Paulo no ano proposto.

\section{MÉTODO}

Estudo epidemiológico, do tipo ecológico, de base populacional. A amostra foi obtida a partir dos casos confirmados de Zika Vírus notificados no Sistema de Informação de Agravos de Notificação com comprovação laboratorial (reverse transcription polymerase chain reaction (RT-PCR)) e/ou clínica epidemiológica, ocorridos nos municípios, distribuídos entre os Grupos de Vigilância Epidemiológica do Estado de São Paulo em 2016.

O Estado de São Paulo é composto por 645 municípios. Para coordenar os Serviços de Vigilância Epidemiológica, o Estado conta, a nível central, com o Centro de Vigilância Epidemiológica uma diretoria técnica, composta por divisões de doenças e agravos à saúde. O Centro de Vigilância Epidemiológica, a nível regional, estrutura-se, atualmente, a partir de 28 Grupos de Vigilância Epidemiológica que compõem todos os municípios do Estado ${ }^{13}$.

Foram selecionadas variáveis a partir da ficha de notificação/conclusão (idade, sexo, raça/cor, escolaridade, município de residência, logradouro, número, zona, classificação final (confirmado), critério de confirmação/ descarte, caso autóctone no município de residência, município, evolução do caso), que é utilizada para Zika Vírus, e para o cálculo da taxa de incidência, a população foi obtida por meio do Instituto Brasileiro de Geografia e Estatística (IBGE) 2016. De posse do banco de dados, com as notificações confirmadas, os casos duplicados foram retirados.

Para a análise dos dados, utilizou-se estatística descritiva, aplicando-se os Softwares TabWin 3.6b, Microsoft
Excel 2016 e o Programa R 3.5.1/RStudio. Além disso, foi aplicada a técnica de georreferenciamento dos casos e, para esse processo, utilizaram-se os campos "logradouro" e "número". Foi realizado o processo automático por meio do site easymapmake e das ferramentas SIGs ArcGis versão 10.5 e Qgis10. A análise espacial foi feita a partir do estimador de densidade Kernel e a estatística de varredura fornecida pelo SaTScan ${ }^{\mathrm{TM}}$ version $9.6 \mathrm{com}$ a incidência dos casos confirmados de Zika Vírus.

A pesquisa foi aprovada pelo Comitê de Ética de Pesquisa da Universidade São Judas, situada no município de São Paulo / SP, sob número de Parecer: 3.167.498. A utilização da base de dados secundária de Zika Vírus 2016, sem identificação dos pacientes, foi autorizada pelo gestor responsável.

\section{RESULTADOS}

Foram notificados 10.449 casos de Zika Vírus no Estado de São Paulo em 2016. Desses, 4.513 (43,2\%) foram confirmados, sendo $4.323(95,8 \%)$ constatados como autóctones. O coeficiente de incidência no Estado foi de 10,1 casos para cada 100.000 habitantes.

A confirmação se deu, na maioria, pelo critério clínico-epidemiológico $(73,3 \%)$. A mediana da idade foi de 33 anos com desvio padrão de 17 . A maioria dos casos ocorreu nos indivíduos do sexo feminino (74,2\%); não gestantes $(78,3 \%)$ e com residência em área urbana $(92,7 \%)$. Em relação às variáveis raça/cor e escolaridade: 44,3\% e $60,6 \%$, respectivamente, não apresentaram informação. Os casos foram confirmados em 141 municípios dos Grupos de Vigilância Epidemiológica (Tabela 1), sendo o maior número no município de Ribeirão Preto (1023) / Grupo de Vigilância Epidemiológica Ribeirão Preto; Barretos (760) / Grupo de Vigilância Epidemiológica Barretos e Jardinópolis (505) / Grupo de Vigilância 
Moura, R.F.; Almeida, A.B.; Análise espacial dos casos confirmados de Zika Vírus no estado de São Paulo, Brasil

Tabela 1 - Distribuição dos Grupos de Vigilância Epidemiológica por número de municipios que confirmaram casos de Zika Virus. Estado de São Paulo, 2016.

\begin{tabular}{|c|c|c|}
\hline Grupo de Vigilância Epidemiológica (GVE) & $\mathbf{n}$ & $\%$ \\
\hline ARAÇATUBA & 7 & 4,96 \\
\hline ARARAQUARA & 12 & 8,51 \\
\hline ASSIS & 3 & 2,13 \\
\hline BARRETOS & 7 & 4,96 \\
\hline BAURU & 4 & 2,84 \\
\hline BOTUCATU & 3 & 2,13 \\
\hline CAMPINAS & 9 & 6,38 \\
\hline CAPITAL & 1 & 0,71 \\
\hline CARAGUATATUBA & 4 & 2,84 \\
\hline FRANCA & 10 & 7,09 \\
\hline FRANCO DA ROCHA & 1 & 0,71 \\
\hline JALES & 7 & 4,96 \\
\hline MARÍLIA & 2 & 1,42 \\
\hline MOGI DAS CRUZES & 4 & 2,84 \\
\hline OSASCO & 3 & 2,13 \\
\hline PIRACICABA & 6 & 4,26 \\
\hline PRESIDENTE VENCESLAU & 1 & 0,71 \\
\hline RIBEIRÃO PRETO & 16 & 11,35 \\
\hline SANTO ANDRÉ & 3 & 2,13 \\
\hline SANTOS & 5 & 3,55 \\
\hline SÃO JOÃO DA BOA VISTA & 6 & 4,26 \\
\hline SÃO JOSÉ DO RIO PRETO & 16 & 11,35 \\
\hline SÃO JOSÉ DOS CAMPOS & 2 & 1,42 \\
\hline SOROCABA & 5 & 3,55 \\
\hline TAUBATÉ & 4 & 2,84 \\
\hline TOTAL & 141 & 100,00 \\
\hline
\end{tabular}

Fonte: SINAN-NET 2016

Figura 1 - Distribuição dos casos confirmados de Zika Vírus, segundo município de residência / Grupo de Vigilância Epidemiológica. Estado de São Paulo, 2016.

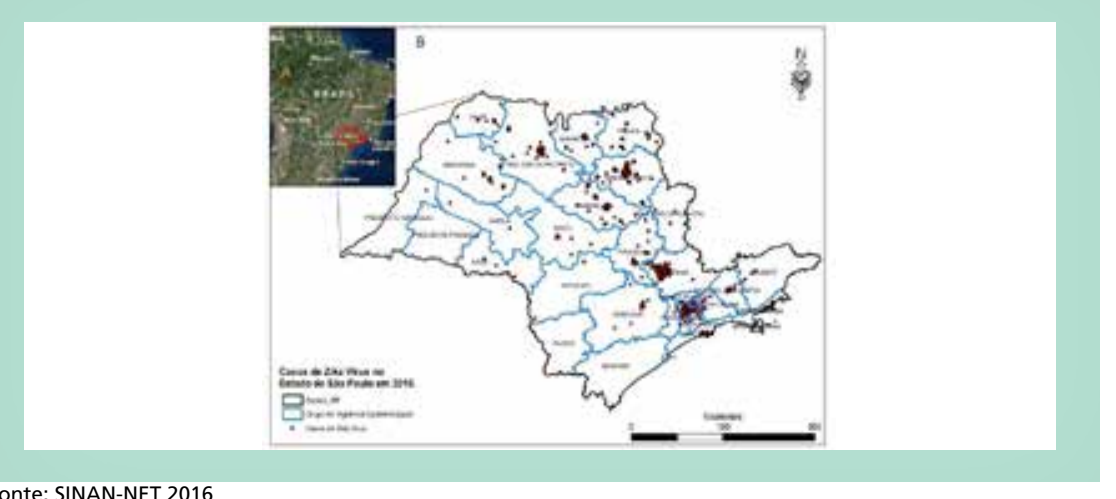

Epidemiológica Ribeirão Preto (Figura 1) e não foram confirmados óbitos.

Os municípios de Jardinópolis / Grupo de Vigilância Epidemiológica Ribeirão Preto; Jaborandi e Barretos / Grupo de Vigilância Epidemiológica Barretos apresentaram maior risco da doença (incidência de 1.192,22; 1.115,94 e 633,61, respectivamente, por 100.000 habitantes) (Figura 2).

A análise de Kernel apontou áreas quentes (densidades dos casos) na região do estudo (Figura 3). Além disso, por meio da estatística de varredura, fornecida pela ferramenta do SaTScan ${ }^{\mathrm{TM}}$, foram identificados os aglomerados dos altos Riscos Relativos dos 645 municípios distribuídos entre os 28 Grupos de Vigilância Epidemiológica do Estado. Os centroides dos 13 aglomerados com valor de " $\mathrm{p}$ " estatisticamente significativos $(p \leq 0,05)$ foram mais intensos nos municípios de Barretos / Grupo de Vigilância Epidemiológica Barretos; Ribeirão Preto / Grupo de Vigilância Epidemiológica Ribeirão Preto; São José do Rio Preto / Grupo de Vigilância Epidemiológica São José do Rio Preto; Pereira Barreto / Grupo de Vigilância Epidemiológica Araçatuba; Alto Alegre e Penápolis / Grupo de Vigilância Epidemiológica Araçatuba; Cravinhos / Grupo de Vigilância Epidemiológica Ribeirão Preto; Gavião Peixoto / Grupo de Vigilância Epidemiológica Araraquara; Jaci / Grupo de Vigilância Epidemiológica São José do Rio Preto; Porto Ferreira / Grupo de Vigilância Epidemiológica Araraquara; Piracicaba / Grupo de Vigilância Epidemiológica Piracicaba; Sumaré e Campinas / Grupo de Vigilância Epidemiológica Campinas com variação do Risco Relativo entre $\geq 2,22$ e $\leq 67,24$ (Figura 4).

\section{DISCUSSÃo}

Observou-se muitos casos confirmados, bem como alta incidência de Zika Vírus no Estado de São Paulo em 2016, comparado com 2015 (82 casos confirmados - Centro de Vigilância Epidemiológica: dados não publica- 
Figura 2 - Distribuição da Taxa de Incidência dos casos confirmados de Zika Virus (por 100000 habitantes), segundo município de residência / Grupo de Vigilância Epidemiológica. Estado de São Paulo, 2016.

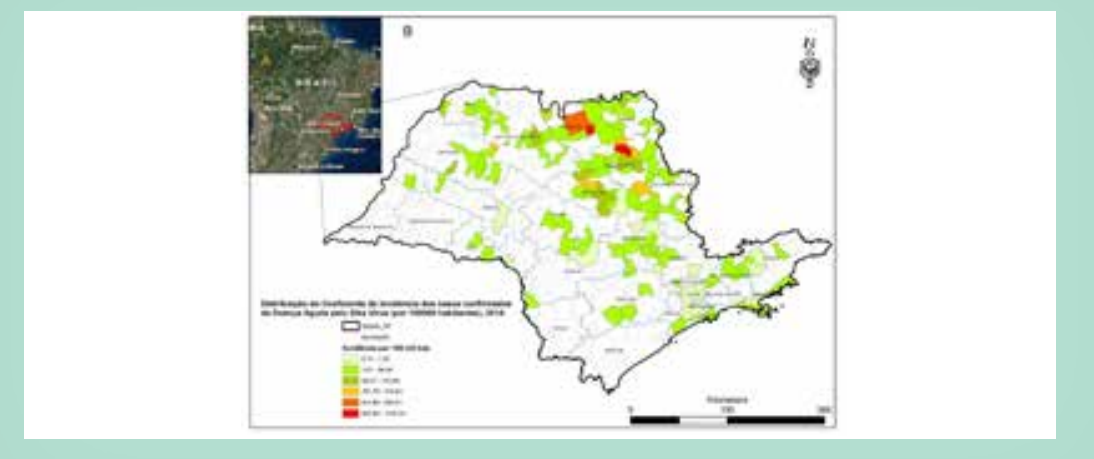

Fonte: SINAN-NET 2016

Figura 3 - Análise de Kernel dos casos confirmados de Zika Virus, segundo municipio de residência / Grupo de Vigilância Epidemiológica. Estado de São Paulo, 2016.

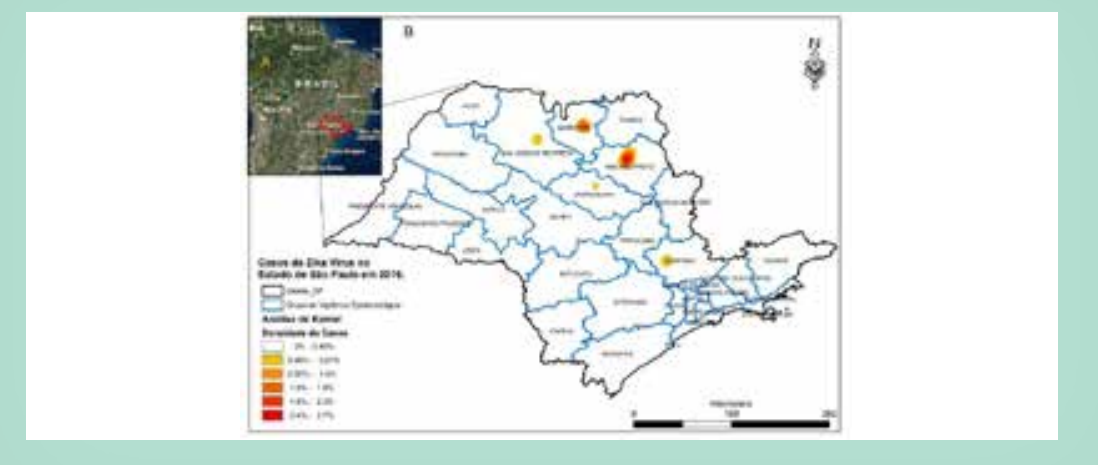

Fonte: SINAN-NET 2016

Figura 4 - Análise de Risco Relativo dos casos confirmados de Zika Vírus, segundo municipio de residência / Grupo de Vigilância Epidemiológica. Estado de São Paulo, 2016.

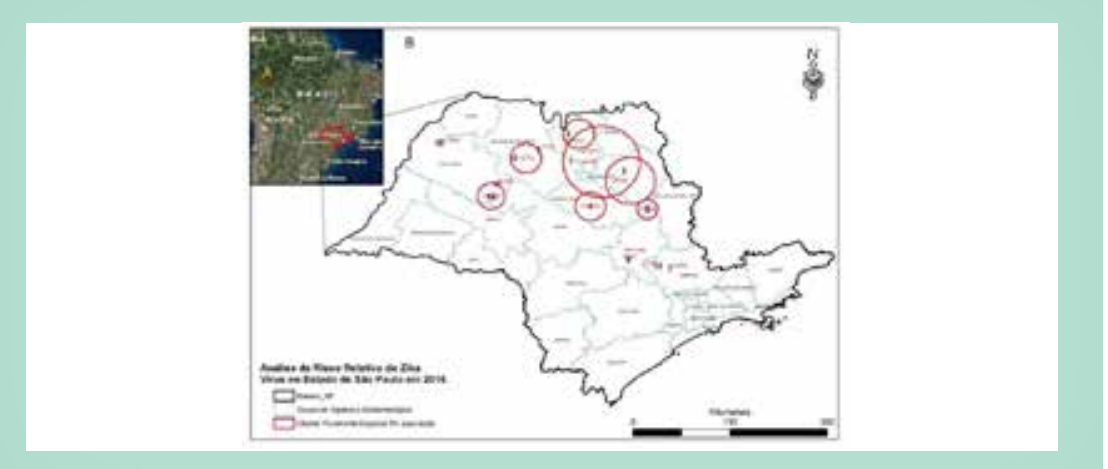

Fonte: SINAN-NET 2016

dos). No entanto, não foram constatados óbitos. No mesmo ano o Ministério da Saúde do Brasil registrou 216.207 casos prováveis de Zika Vírus e confirmou, laboratorialmente, oito óbitos, sendo quatro no Rio de Janeiro, dois no Espírito Santo, um no Maranhão e um na Paraíba ${ }^{14}$.

A análise descritiva constatou que todos os indivíduos do Estado de São
Paulo apresentavam vulnerabilidade para o Zika Vírus. Torna-se importante ressaltar que o Vírus Zika afeta a todos os grupos etários e ambos os sexos e que todos os Estados e Distrito Federal do Brasil apresentaram risco para o Zika Vírus ${ }^{15}$.

No tangente ao sexo, observou-se a prevalência à maioria dos casos confirmados em mulheres com idade economicamente ativa. Esse é um padrão já relatado em diversas pesquisas, ou seja, afirmam que esse grupo é o mais afeta$\mathrm{do}^{16-18}$. Esse resultado é sugestivo que, em geral, as mulheres que permanecem mais tempo em contato com o ambiente doméstico são mais susceptíveis ao Zika Vírus.

A análise da distribuição espacial do Zika Vírus possibilitou determinar o padrão da situação desse agravo à saúde no Estado de São Paulo, evidenciando disparidades espaciais que levou a delimitação da área de risco.

Verificou-se que a distribuição espacial do Zika Vírus no Estado de São Paulo seguiu um padrão heterogêneo, pois, os focos tenderam-se a agregar-se em áreas específicas. Além disso, os municípios das regiões norte e noroeste apresentaram crescente Risco Relativo.

A despeito desse resultado, Costa et al. ${ }^{19}$ realizaram um estudo sobre análise espacial de casos prováveis de dengue, chikungunya e Zika Vírus no Estado do Maranhão no Brasil e verificaram que nos anos de 2015 e 2016 a distribuição dos casos de Zika Vírus foi homogênea. Além disso, mostrou um padrão espacial significativo de Zika Vírus nas regiões oeste e norte do Estado.

Com relação aos possíveis fatores explicativos para a distribuição de Zika Vírus, a densidade populacional mostrou-se relevante na análise espacial. Segundo Lima-Camara ${ }^{20}$, a densidade populacional é considerada um fator fundamental para as altas taxas de incidência de arboviroses, uma vez que ambientes mais populosos favorecem a proliferação do vetor devido às condi- 
ções ideais para a sua reprodução, bem como ao maior número de indivíduos suscetíveis para novas infecções.

Os mosquitos Aedes Aegypti, geralmente, não vivem em altitudes acima de 2000 metros (6500 pés) devido às condições ambientais. No entanto, a maioria dos territórios brasileiros é baixa. Assim, esses fatores podem contribuir concomitantemente para a ampla distribuição desse mosquito e doença na maior parte do Estado de São Paulo e Brasil ${ }^{21}$.

Na Colombia, em 2016, a maioria dos casos relatados ocorreu principalmente em áreas de baixa altitude, e pontos quentes persistentes foram observados. Taxas de infecção mais altas foram relatadas na parte nordeste da área de estudo 22
Finalmente, este trabalho apresentou limitações devido a utilização de dados secundários que, podem apresentar inconsistência em relação à quantidade, qualidade e processamento das informações. E como ponto forte, ao analisar a espacialidade da incidência do Zika Vírus no Estado de São Paulo ficou evidenciado sua estreita relação com as áreas de maior presença do vetor.

\section{CONCLUSÃO}

O potencial de epidemias em determinados Municípios do Estado de São Paulo é particularmente preocupante, dado ao grande número de pessoas potencialmente suscetíveis ao Zika Vírus.

Os resultados deste estudo oferecem informações fundamentais para auxiliar os gestores em saúde pública na tomada de decisões, sensível ao tempo, nos níveis municipal e estadual.

O conhecimento de áreas de potencial preocupação interfere no planejamento prévio e reuni recursos suficientes que são essenciais para a formulação de programas de saúde pública bem-sucedidos.

Ações preventivas devem ser tomadas para a eliminação do mosquito e diminuir a transmissão e os casos do Zika Vírus, bem como outras arboviroses, como dengue, chikungunya e febre amarela, principalmente nos locais apontados pelo estudo.

O uso de mapas de risco pode ajudar a orientar decisões para a prevenção e controle do Zika Vírus.

\section{Referências}

1. Ferraris $P, Y$ ssel $H$, Missé D. Zika virus infection: an update. Microbes Infect. 2019;21(8-9):353-360.

2. Proenca-Modena JL, Milanez GP, Costa ML, Judice CC, Maranhão Costa FT. Zika virus: lessons learned in Brazil. Microbes Infect. 2018;20(1112):661-669.

3. Luz KG, Santos GIV, Vieira RM. Zika Virus Fever. Epidemiol. Serv Saúde 2015; 24(4):785-788.

4. Campos GS, Bandeira AC, Sardi SI. Zika virus outbreak, Bahia, Brazil [letter]. Emerging Infectious Diseases 2015;21(10):1885-1886.

5. Musso D, DJ Gubler. Vírus Zika. Clin Microbiol Rev. 2016;29 (3): 487-524.

6. Nunes MRT, Faria NR, de Vasconcelos JM, Golding N, Kraemer MVG, de Oliveira LF. Emergence and potential for spread of Chikungunya virus in Brazil. BMC Med 2015;13(1):102.

7. Baud D, Gubler DJ, Schaub B. Lanteri MC, Musso D. An update on Zika virus infection. Lancet 2017; 390:2099-20109.

8. Rouquayrol MZ, Gurgel M. Epidemiologia \& Saúde. 8. ed. Rio de Janeiro: MedBook, 2017.

9. Romero Canal M, da Silva Ferreira ER, Estofolete CF, Martiniano Dias A, Tukasan C, Bertoque AC et al. Spatiotemporal-based clusters as a method for dengue surveillance. Rev Panam Salud Publica. 2017;41:e162.

10. Aguiar BS, Lorenz C, Virginio F, Suesdek L, Chiaravalloti-Neto F. Potential Risks of Zika and Chikungunya Outbreaks in Brazil: a Modelling Study. International Journal of Infectious Diseases. 2018;70:20-29.

11. Brasil. Ministério da Saúde. Secretaria de Vigilância em Saúde. Programa Nacional de Controle da Dengue. Protocolo para implantação de unidades sentinelas para Zika vírus. Brasília: Ministério da Saúde; 2016.

12. Brasil. Ministério da Saúde. Portaria $n^{\circ} 204$ de 17 de fevereiro de 2016. Define a Lista Nacional de Notificação Compulsória de doenças, agravos e eventos de saúde pública nos serviços de saúde públicos e privados em todo o território nacional, nos termos do anexo, e dá outras providências. Diário Oficial da União 2016. 18 fev.

13. São Paulo (Estado). Secretaria do Estado da Saúde. Centro de Vigilância Epidemiológica "Prof. Alexandre Vranjac". Sobre o CVE. [Internet]. [cited 2018 set. 19]. São Paulo: 2018. Available from: http://www.saude.sp.gov. br/cve-centro-de-vigilancia-epidemiologica-prof.-alexandre-vranjac/institucional/sobre-o-cve>.

14. Brasil. Ministério da Saúde. Secretaria de Vigilância em Saúde. Boletim Epidemiológico. Brasília: Ministério da Saúde 2017; 48(29).

15. Brasil. Ministério da Saúde. Secretaria de Vigilância em Saúde. Departamento de Vigilância das Doenças Transmissíveis. Protocolo de vigilância e resposta à ocorrência de microcefalia relacionada à infecção pelo Vírus Zika. Brasília: Ministério da Saúde, 2015.

16. Brasil P, Calvet GA, Siqueira AM, Wakimoto M, Sequeira PC, Nobre A et al. Zika Virus Outbreak in Rio de Janeiro, Brazil: Clinical Characterization, Epidemiological and Virological Aspects. PLOS Neglected Tropical Diseases [Internet]. [cited 2018 set. 17]. Brasil: 2016. Available from: https://journals. plos.org/plosntds/article/file?id=10.1371/journal.pntd.0004636\&type=printable

17. Brito CAA, Brito $C C M$, Oliveira AC, Rocha $M$, Atanásio $C$, Asfora $C$ et al. Zika in Pernambuco: rewrinting the first outbreak. Revista da Sociedade Brasileira de Medicina Tropical 2016; 49(5):553-558.

18. Cardoso CW, Paploski IA, Kikuti M, Rodrigues MS, Silva MM, Campos GS et al. Outbreak of Exanthematous IIIness Associated with Zika, Chikungunya, and Dengue Viruses, Salvador, Brazil. Emerging Infections Diseases 2015;21(12):2274-2276.

19. Costa SSB, Branco MRFC, Aquino Junior J, Rodrigues ZMR, Queiroz RCS Araujo AS et al. Spatial analysis of probable cases of dengue fever, chikungunya fever and zika virus infections in Maranhao State, Brazil. Rev Inst Med Trop Sao Paulo. 2018; 60:e62.

20. Lima-Camara TN. Arboviroses emergentes e novos desafios para a saúde pública no Brasil. Rev. Saúde Pública. 2016;50:36.

21. Centers for Disease Control and Prevention (CDC). Chikungunya virus - symptoms, diagnosis, \& treatment. 2016. [Internet]. [cited 2016 out. 22]. 2016. Available from: https://www.cdc. gov/chikungunya/symptoms/index. html.

22. Rodriguez-Morales AJ, Haque U, Ball JD, García-Loaiza CJ, Galindo-Marquez ML, Sabogal-Roman JA et al. Spatial distribution of Zika virus infection in Northeastern Colombia. Infez Med. 2017; 25(3):241-246. 